\title{
An Online System of Multispectral Palmprint Verification
}

\author{
David Zhang, Fellow, IEEE, Zhenhua Guo, Guangming Lu, Lei Zhang, Member, IEEE, and Wangmeng Zuo
}

\begin{abstract}
Palmprint is a unique and reliable biometric characteristic with high usability. With the increasing demand of highly accurate and robust palmprint authentication system, multispectral imaging has been employed to acquire more discriminative information and increase the antispoof capability of palmprint. This paper presents an online multispectral palmprint system that could meet the requirement of real-time application. A data acquisition device is designed to capture the palmprint images under Blue, Green, Red, and near-infrared (NIR) illuminations in less than $1 \mathrm{~s}$. A large multispectral palmprint database is then established to investigate the recognition performance of each spectral band. Our experimental results show that the Red channel achieves the best result, whereas the Blue and Green channels have comparable performance but are slightly inferior to the NIR channel. After analyzing the extracted features from different bands, we propose a score level fusion scheme to integrate the multispectral information. The palmprint verification experiments demonstrated the superiority of multispectral fusion to each single spectrum, which results in both higher verification accuracy and antispoofing capability.
\end{abstract}

Index Terms-Biometrics, multispectral, orientation code, palmprint verification, score level fusion.

\section{INTRODUCTION}

B IOMETRICS refers to the study of methods for recognizing humans based on one or more physical or behavioral traits $[1,2]$. As an important biometrics characteristic, palmprint has been attracting much attention [3]-[14] because it has merits, such as high speed, user friendliness, low cost, and high accuracy. However, there is room for improvement of online palmprint systems in the aspects of accuracy and capability of spoof attacks [26]. Although 3-D imaging could be used to address these issues, the expensive and bulky device makes it difficult to be applied for real applications. One solution

Manuscript received March 7, 2009; revised July 6, 2009. First published October 16, 2009; current version published January 7, 2010. This work was supported in part by the General Research Fund from the HKSAR Government, by the Central Fund from The Hong Kong Polytechnic University, by the Natural Scientific Research Innovation Foundation, Harbin Institute of Technology, by the Key Laboratory of Network Oriented Intelligent Computation (Shenzhen), by the Natural Science Foundation of China under Grant 60620160097 and Grant 60803090, and by the 863 under Grant 2006AA01Z193. The Associate Editor coordinating the review process for this paper was Dr. Salvatore Baglio.

D. Zhang, Z. Guo, and L. Zhang are with the Biometrics Research Centre, Department of Computing, The Hong Kong Polytechnic University, Kowloon, Hong Kong (e-mail: csdzhang@comp.polyu.edu.hk).

G. Lu is with the Biocomputing Research Center, Shenzhen Graduate School, Harbin Institute of Technology, Shenzhen 518055, China.

W. Zuo is with the Biocomputing Research Center, School of Computer Science and Technology, Harbin Institute of Technology, Harbin 150001, China.

Color versions of one or more of the figures in this paper are available online at http://ieeexplore.ieee.org.

Digital Object Identifier 10.1109/TIM.2009.2028772 to these problems can be multispectral imaging [15]-[17], which captures an image in a variety of spectral bands. Each spectral band highlights specific features of the palm, making it possible to collect more information to improve the accuracy and antispoofing capability of palmprint systems.

Multispectral analysis has been used in palm-related authentication [18]-[21], [33]. Rowe et al. [19] proposed a multispectral whole-hand biometric system. The object of this system was to collect palmprint information with clear fingerprint features, and the imaging resolution was set to 500 dpi. However, the low speed of feature extraction and feature matching makes it unsuitable for real-time applications. Likforman-Sulem et al. [20] used multispectral images in a multimodal authentication system; however, their system used an optical desktop scanner and a thermal camera, which make the system very costly. The imaging resolution is also too high (600 dpi, the FBI fingerprint standard) to meet the real-time requirement in practical biometric systems. Wang et al. [21] proposed a palmprint and palm vein fusion system that could simultaneously acquire two kinds of images. The system uses one color camera and one nearinfrared (NIR) camera and requires a registration procedure of about 9 s. Hao et al. [18], [33] developed a contact-free multispectral palm sensor. However, the image quality is limited, and hence, the recognition accuracy is not very high. Overall, multispectral palmprint scanning is a relatively new topic, and the aforementioned works stand for the state-of-the-art work.

The information presented by multiple biometric measures can be consolidated at four levels: 1) image level; 2) feature level; 3) matching score level; and 4) decision level [25]. Wang et al. [21] fused palmprint and palm vein images by using a novel edge-preserving and contrast-enhancing wavelet fusion method for use of the personal recognition system. Some good results in accuracy were reported, but the image registration procedure in it takes $9 \mathrm{~s}$, which hinders it from real-time implementation. Hao et al. [18] evaluated several well-known image-level fusion schemes for multispectral palm images. However, their data set was too small (84 images) to be conclusive. In [33], Hao et al. extended their work to a larger database and proposed a new feature-level registration method for image fusion. The results by various image fusion methods were also improved. Although image- and feature-level fusion can integrate the information provided by each spectral band, the required registration procedure is often too time consuming [21]. As to matching score fusion and decision level fusion, it has been found [25] that the former works better than the latter because match scores contain more information about the input pattern, and it is easy to access and combine the scores generated by different matchers. For these reasons, information 


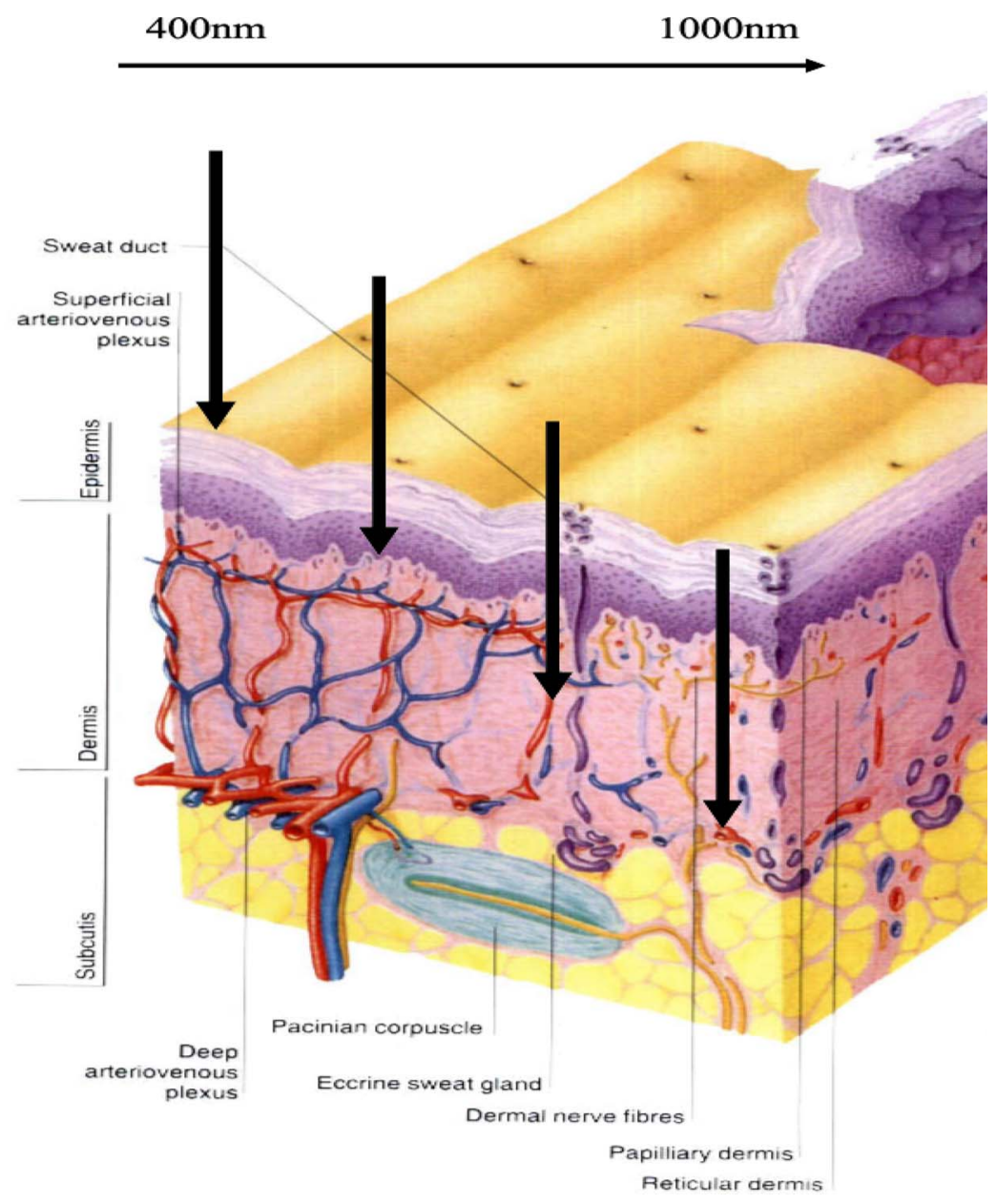

Fig. 1. Cross-sectional anatomy of the skin.

fusion at score level is the most commonly used approach in multimodal biometric systems and multispectral palmprint systems [19], [20].

In this paper, we propose a low-cost multispectral palmprint system that can operate in real time and acquire high-quality images. The proposed system collects palmprint images in the visible and NIR spectra. Compared with traditional palmprint recognition approaches, the developed system can improve much the recognition accuracy by fusing the information provided by multispectral palmprint images at the score level. On the other hand, by utilizing the correlation between spectra, the system is robust to antispoof attack.

The rest of this paper is organized as follows: Section II describes the developed online multispectral palmprint system. Section III introduces the multispectral palmprint analysis methodology. Section IV reports our experimental results in a large multispectral palmprint database. Section $\mathrm{V}$ gives the conclusions and future work.

\section{Online Multispectral Palmprint System Design}

In this section, we describe the components of our proposed system and its parameters. The two basic considerations in the design of a multispectral palmprint system are the following: 1) the color-absorptive and color-reflective characteristics of human skin and 2) the light spectra to be used when acquiring images. Human skin is made up of three layers: 1) epidermis; 2) dermis; and 3) subcutis, as shown in Fig. 1. Each layer will contain a different proportion of blood and fat. The epidermis also contains melanin, whereas the subcutis contains veins [22]. Different light wavelengths will penetrate to different skin layers and illuminate in different spectra. NIR light penetrates human tissue further than visible light, and blood absorbs more NIR energy than the surrounding tissue (e.g., fat or melanin) [23]. Our system acquires spectral information from all three dermal layers by using both visible and NIR bands. In the visible spectrum, a three-monocolor LED array is used with Red peaking at $660 \mathrm{~nm}$, Green peaking at $525 \mathrm{~nm}$, and Blue peaking at $470 \mathrm{~nm}$. In the NIR spectrum, an NIR LED array peaking at $880 \mathrm{~nm}$ is used. It has been shown that light in the 700- to $1000-\mathrm{nm}$ range can penetrate human skin, whereas $880-930 \mathrm{~nm}$ provides a good contrast of subcutaneous veins [23].

Fig. 2 shows the structure of the designed multispectral palmprint image acquisition device, and Fig. 3 shows the prototype of our device. It consists of a charge-coupled device (CCD) camera, lens, an A/D converter, a multispectral light source, and a light controller. A monochromatic CCD is placed at the bottom of the device. The A/D converter connects the CCD and the computer. The light controller is used to control the multispectral light. 


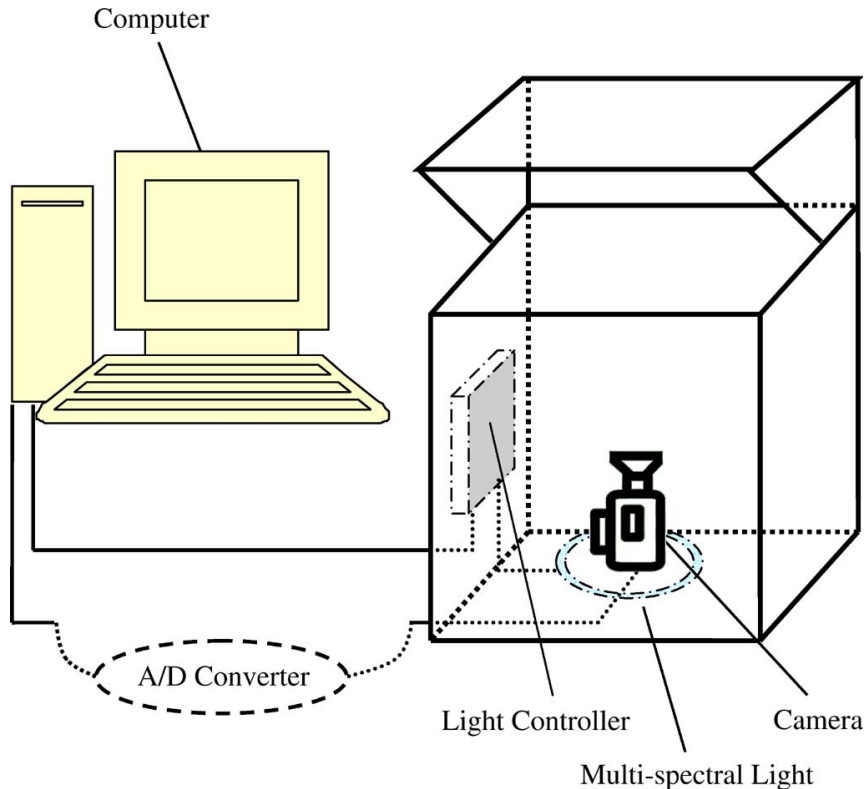

Fig. 2. Structure of the multispectral palmprint acquisition device.

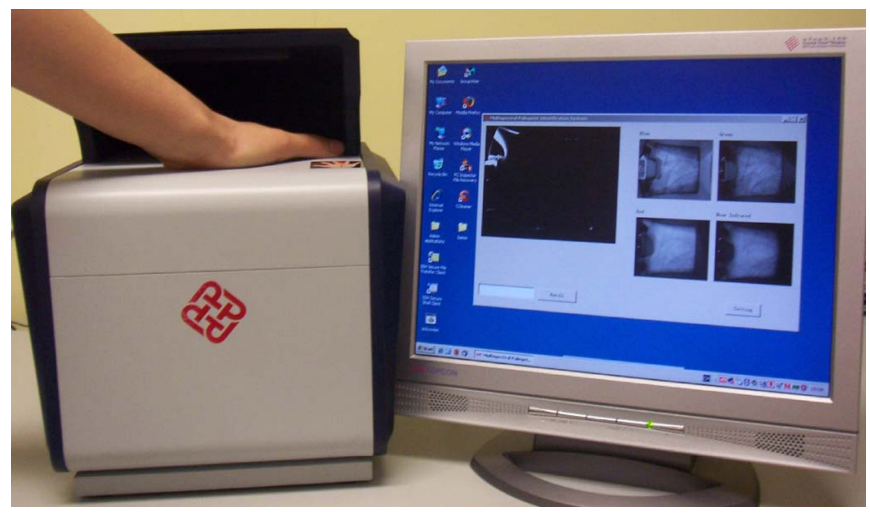

Fig. 3. Prototype of the proposed multispectral palmprint system.

The system can capture palmprint images in a resolution of either $352 \times 288$ or $704 \times 576$. A user is asked to put his/her palm on the platform. Several pegs serve as control points for the placement of the user's hands. Four palmprint images of the palm are collected under different spectral lights. The switching time between the two consecutive lights is very short, and the four images can be captured in a very short time $(<1 \mathrm{~s})$.

Fig. 4 shows a typical multispectral palmprint sample in the a) Blue, b) Green, c) Red, and d) NIR bands. It can be observed that the line features are clearer in the Blue and Green bands than in the Red and NIR bands. While the Red band can reveal some vein structure, the NIR band can show the palm vein structures as well as partial line information. In our system, the palm vein structure acquired in the NIR band is not as clear as that reported in [23], because the CCD in our system is a standard closed-circuit television camera, instead of a NIRsensitive camera, to reduce cost. Moreover, we do not add an infrared filter in front of the CCD because the infrared filter would cut off the visible light and affect the acquisition of clear palmprint images under the visible spectrum.

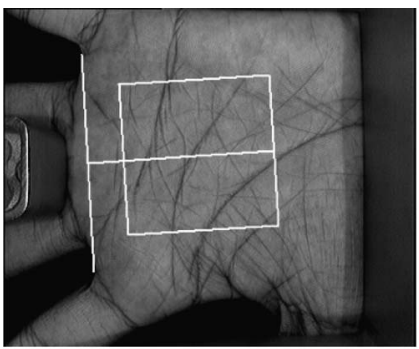

(a)

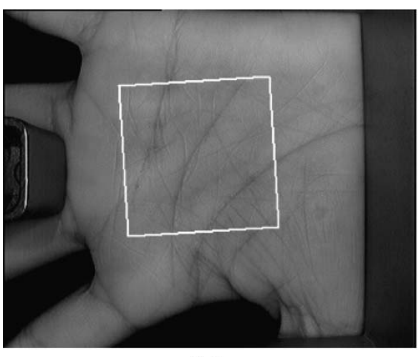

(c)

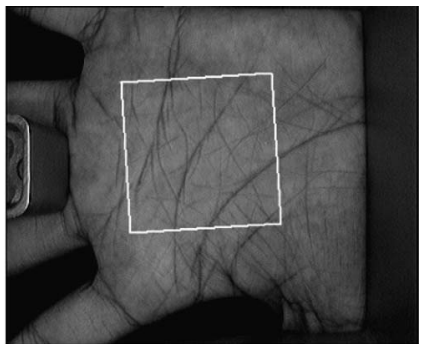

(b)

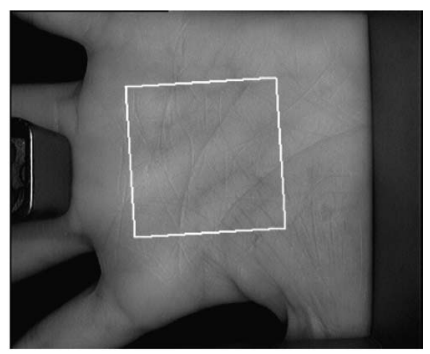

(d)
Fig. 4. Typical multispectral palmprint sample. (a) Blue. (b) Green. (c) Red. (d) NIR. The white square is the ROI of the image.

A region of interest (ROI) will be extracted from the palmprint image for further feature extraction and matching. This can reduce the data amount in feature extraction and matching and reduce the influence of rotation and translation of the palm. In this paper, the ROI extraction algorithm in [8] is used and applied to the Blue band to find the ROI coordinate system. After ROI extraction, the translation or rotation is usually small between two images. Thus, no more registration procedure is necessary. Fig. 4 shows the ROI of the palmprint image, and Fig. 5 shows the cropped ROI images.

After obtaining the ROI for each band, feature extraction and matching will be applied to these ROI images. The final verification decision will be made by score-level fusion of all bands. Fig. 6 shows the framework of the proposed online verification system.

\section{Multispectral Palmprint Image Analysis}

In this section, we first describe how features are extracted and matched for each spectrum band of multispectral palmprint images and then analyze the correlation between the spectral bands. Finally, a score-level fusion scheme is presented for decision making.

\section{A. Feature Extraction and Matching for Each Band}

There are mainly three kinds of algorithms currently used in palmprint authentication: 1) subspace learning [3]-[5]; 2) line detection [6], [7]; and 3) texture-based coding [8]-[11]. The online system usually uses texture-based coding because it has high accuracy and is robust to illumination and fast for matching. The proposed system employs orientation-based coding [10], which is a state-of-the-art texture-based coding algorithm, for feature extraction. 


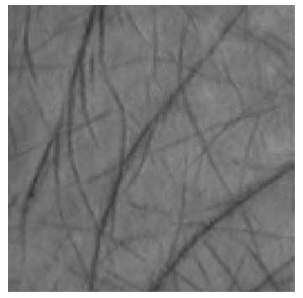

(a)

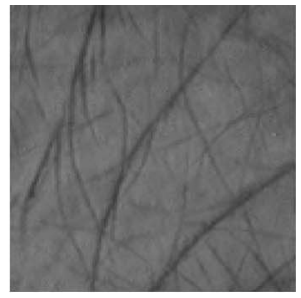

(b)

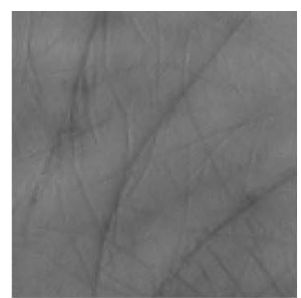

(c)

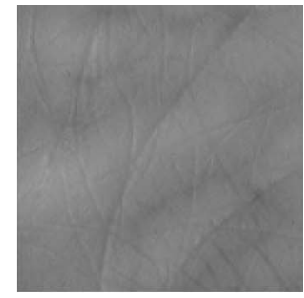

(d)

Fig. 5. ROI of Fig. 4. (a) Blue. (b) Green. (c) Red. (d) NIR.

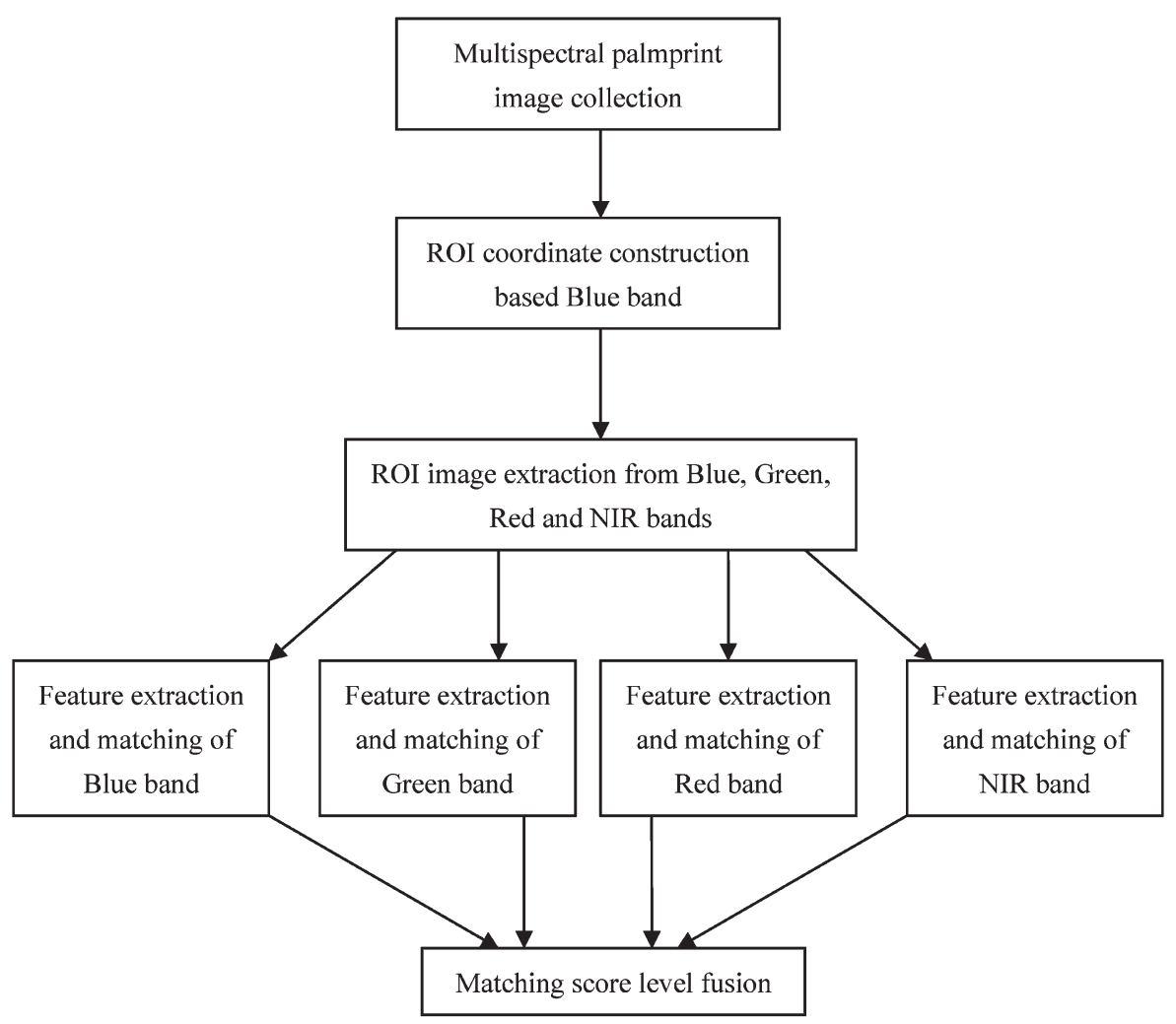

Fig. 6. Framework of the online verification system.

By viewing the line features in palmprint images as negative lines, we apply six Gabor filters along different directions $\left(\theta_{j}=j \pi / 6\right.$, where $\left.j=\{0,1,2,3,4,5\}\right)$ to the palmprint images for orientation feature extraction. For each pixel, the orientation corresponding to the minimal response is taken as the feature at this pixel [10]. The employed Gabor filter is

$$
\psi(x, y, \omega, \theta)=\frac{\omega}{\sqrt{2 \pi \kappa}} e^{-\frac{\omega^{2}}{8 \kappa^{2}}\left(4 x^{\prime 2}+y^{\prime 2}\right)}\left(e^{i \omega x^{\prime}}-e^{-\frac{\kappa^{2}}{2}}\right)
$$

where $x^{\prime}=\left(x-x_{0}\right) \cos \theta+\left(y-y_{0}\right) \sin \theta, y^{\prime}=-\left(x-x_{0}\right) \sin \theta+$ $\left(y-y_{0}\right) \cos \theta,\left(x_{0}, y_{0}\right)$ is the center of the function, $\omega$ is the radial frequency in radians per unit length, $\theta$ is the orientation of the Gabor functions in radians, $\kappa=\sqrt{2 \ln 2}\left(\left(2^{\phi}+1\right) /\left(2^{\phi}-\right.\right.$ $1)$ ), and $\phi$ is the half-amplitude bandwidth of the frequency response. To reduce the influence of illumination, the direct current is removed from the filter. Fig. 7 shows some feature maps extracted from Fig. 5 using a variety of parameters.

Since there are totally six different orientations, we can code them by using 3 bits, as listed in Table I. This coding scheme is to make the bit-wise difference proportional to the angular dif- ference [10]. Therefore, the difference between two orientation maps could be measured by using the Hamming distance as

$$
D(P, Q)=\frac{\sum_{y=0}^{M} \sum_{x=0}^{N} \sum_{i=1}^{3}\left(P_{i}^{b}(x, y) \otimes Q_{i}^{b}(x, y)\right)}{3 M * N}
$$

where $P$ and $Q$ represent two palmprint orientation feature maps, and $P_{i}^{b}$ and $Q_{i}^{b}$ are the $i$ th bit plane of $P$ and $Q$, respectively. The symbol " $\otimes$ " represents bitwise exclusive OR. Obviously, $D$ is between 0 and 1 , and for perfect matching, the distance will be 0 .

To further reduce the influence of imperfect ROI extraction, in matching, we translate one of the two feature maps vertically and horizontally from -3 to 3 . The minimal distance obtained by translated matching is treated as the final distance.

\section{B. Interspectral Correlation Analysis}

To remove the redundant information of multispectral images, we investigate the correlation between different bands in a quantitative way. By using the Gabor filter in (1), palmprint 


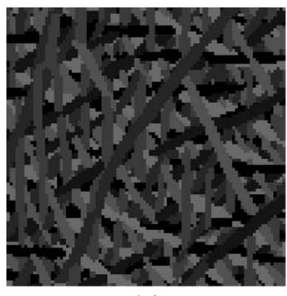

(a)

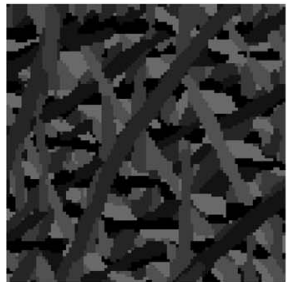

(b)

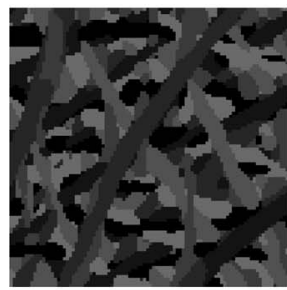

(c)

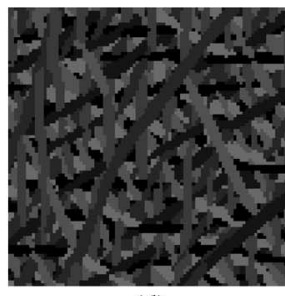

(d)

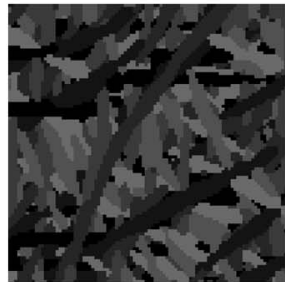

(e)

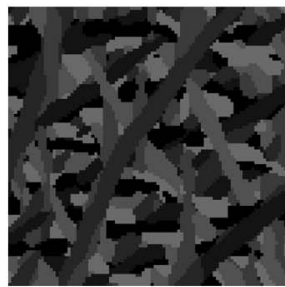

(f)

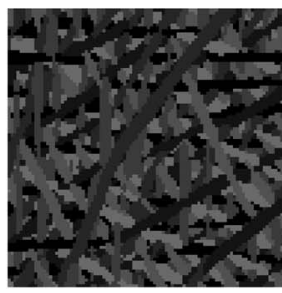

$(\mathrm{g})$

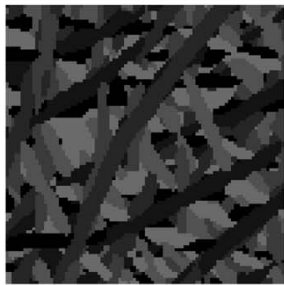

(h)

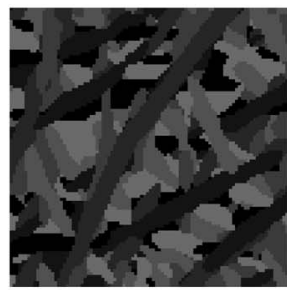

(i)

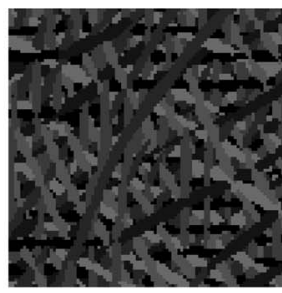

(j)

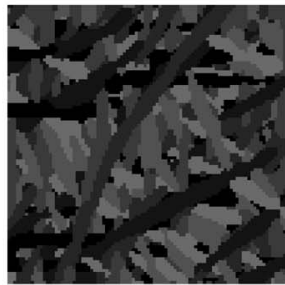

$(\mathrm{k})$

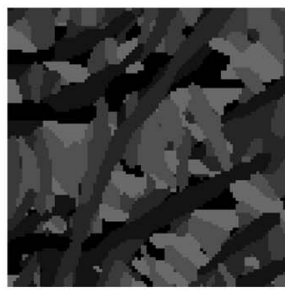

(1)

Fig. 7. Feature map extracted from Fig. 5. Different gray values represent different orientation features. The three maps in each column from left to right are all extracted from the same ROI but using three different parameters. Thus, (a)-(c) are extracted from Blue, (d)-(f) are extracted from Green, and (g)-(i) are extracted from Red. The maps in the fourth column (j)-(1) are extracted from NIR. The same setting is used for each row.

TABLE I

BIT REPRESENTATION OF THE ORIENTATION CODING

\begin{tabular}{cccc}
\hline \hline Orientation Value & Bit 0 & Bit 1 & Bit 2 \\
\hline 0 & 0 & 0 & 0 \\
$\pi / 6$ & 0 & 0 & 1 \\
$\pi / 3$ & 0 & 1 & 1 \\
$\pi / 2$ & 1 & 1 & 1 \\
$2 \pi / 3$ & 1 & 1 & 0 \\
$5 \pi / 6$ & 1 & 0 & 0 \\
\hline
\end{tabular}

TABLE II

Statistic of InTERspectral Distance

\begin{tabular}{ccccc}
\hline $\begin{array}{c}\text { Mean/ } \\
\text { Minimal/ } \\
\text { Maximal of Distance }\end{array}$ & Blue & Green & Red & NIR \\
\hline Blue & 0 & $0.1571 /$ & $0.3110 /$ & $0.3910 /$ \\
& & $0.0915 /$ & $0.2083 /$ & $0.2884 /$ \\
Green & 0.3441 & 0.4420 & 0.4801 \\
& & $0.3030 /$ & $0.3840 /$ \\
Red & & $0.2002 /$ & $0.2920 /$ \\
NIR & & 0.4486 & 0.4650 \\
& & & 0 & $0.2566 /$ \\
& & & $0.1523 /$ \\
\hline
\end{tabular}

features are individually extracted for each spectral band, and then the interspectral distance is computed by (2) for the same palm. Table II shows the statistics of interspectral distance, and Table III shows that of intraspectral distance. From Table II, we can see that as the difference between spectra increases, the feature difference increases. For example, the average difference between Blue and Green is 0.1571, whereas that of Blue and NIR is 0.3910 . This indicates that different spectra
TABLE III

STATISTIC OF INTR ASPECTRAL DISTANCE

\begin{tabular}{ccc}
\hline Spectrum & Mean of Genuine & Mean of Impostor \\
\hline Blue & 0.2600 & 0.4621 \\
Green & 0.2686 & 0.4643 \\
Red & 0.2143 & 0.4561 \\
NIR & 0.2511 & 0.4627 \\
\hline \hline
\end{tabular}

can be used to highlight different textural components of the palm. Meanwhile, compared with the impostor distance, which can be assumed to be independent and close to 0.5 [28], the interspectral distance is much smaller, which shows that different spectral bands are correlated rather than independent.

\section{Score Level Fusion Scheme}

Generally, the more information is used, the better performance could be achieved. However, since there is some overlapping of the discriminating information between different bands, a simple summing of the matching scores of all the bands may not improve much the final accuracy. Suppose there are $k$ kinds of features $\left(F_{i}^{X}, i=\{1,2, \ldots, k\}\right)$. For two samples $X$ and $Y$, the distance using the simple sum rule is defined as

$$
d_{\text {Sum }}(X, Y)=\sum_{i=1}^{k} d\left(F_{i}^{X}, F_{i}^{Y}\right)
$$

where $d\left(F_{i}^{X}, F_{i}^{Y}\right)$ is the distance for the $i$ th feature.

Fig. 8 shows an example of score-level fusion by summation. There are two kinds of features $\left(F_{i}^{X}, i=\{1,2\}\right)$ for three samples $\left\{X_{1}, X_{2}, Y_{1}\right\}$, where $X_{1}$ and $X_{2}$ belong to the same 


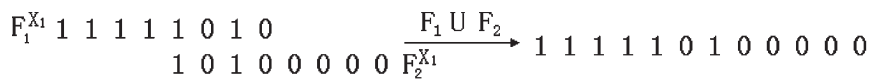

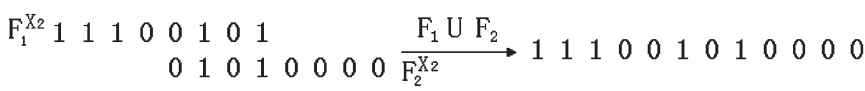

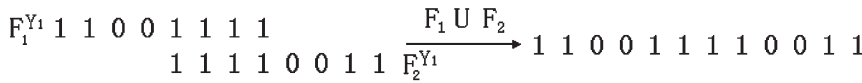

Fig. 8. Example of sum score fusion.

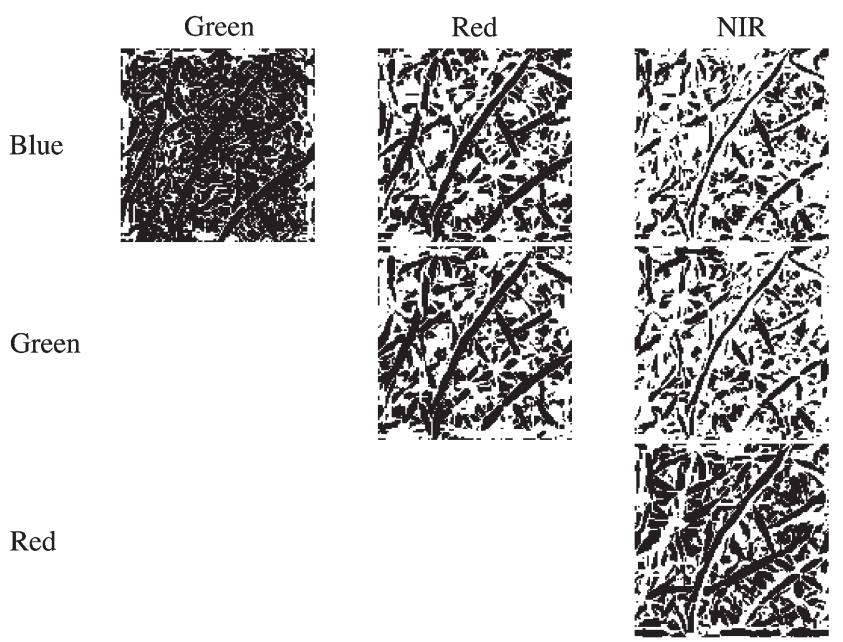

Fig. 9. Overlapping features between different spectra. (Black pixels represent overlapping features, whereas white pixels represent nonoverlapping features.)

class, and $Y_{1}$ belongs to another class. By (3), we can get $d_{S u m}\left(X_{1}, X_{2}\right)=9$, and $d_{S u m}\left(X_{1}, Y_{1}\right)=8$. In fact, the true distances between $X_{1}$ and $X_{2}$, and $X_{1}$ and $Y_{1}$ without information overlapping should be 5 and 6 , respectively. Because there is an overlapping part between the two features, it will be counted twice by using the sum rule [see (3)]. Sometime, such kind of overcomputing may make the simple score-level fusion fail, as shown in the foregoing example. For multispectral palmprint images, most of the overlapping features between two spectral bands locate on the principal lines, as shown in Fig. 9. By using the sum rule [see (3)], those line features will be overcounted so that it may fail to classify two palms with similar principal lines.

From the preceding analysis, we can see that if we can find a score-level fusion strategy to reduce the overlapping effect, better verification result can be expected. The "U" operator in the set theory gives us a good hint, which is defined as

$$
X \cup Y=X+Y-X \cap Y \text {. }
$$

Based on (4), we define a score-level fusion rule that tends to minimize the overlapping effect on the fused score as

$$
\begin{aligned}
d_{F_{1} \cup F_{2}}(X, Y)= & d\left(F_{1}\right)+d\left(F_{2}\right)-d\left(F_{1} \cap F_{2}\right) \\
= & d\left(F_{1}^{X}, F_{1}^{Y}\right)+d\left(F_{2}^{X}, F_{2}^{Y}\right) \\
& -\frac{\left(d\left(F_{1}^{X}, F_{1}^{Y}\right)+d\left(F_{2}^{X}, F_{2}^{Y}\right)\right)}{2} \\
& * P_{\mathrm{OP}}\left(F_{1}, F_{2}\right)
\end{aligned}
$$

TABLE IV

Statistical Percentage (in Percent) of Overlapping Features BETWEEN AND AMONG DifFERENT SPECTRAL BANDS ON HALF OF THE DATABASE

\begin{tabular}{ccc}
\hline \hline Spectra & Mean Percentage & Standard Percentage \\
Blue and Green & 72.6535 & 3.9052 \\
Blue and Red & 47.0632 & 4.9618 \\
Blue and NIR & 33.6842 & 5.0552 \\
Green and Red & 47.3961 & 4.3286 \\
Green and NIR & 34.3067 & 4.6105 \\
Red and NIR & 55.2617 & 5.5367 \\
Blue, Green and Red & 38.8421 & 4.6939 \\
Blue, Green and NIR & 26.9385 & 4.3295 \\
Blue, Red and NIR & 26.3367 & 4.3802 \\
Green, Red and NIR & 26.8390 & 4.2041 \\
Blue, Green, Red and NIR & 21.9879 & 3.9503 \\
\hline
\end{tabular}

where $P_{\mathrm{OP}}\left(F_{1}, F_{2}\right)$ is the overlapping percentage between two feature maps. Here, two assumptions are made. First, we assume that the overlapping percentage of two feature maps is nearly the same for different palms. There are two reasons for us to make this assumption. One reason is that the difference of overlapping percentage between different palms is relatively small, as can be seen in Table IV. The other reason is that although $P_{\mathrm{OP}}\left(F_{1}, F_{2}\right)$ can be computed for any given two feature maps, it will spend some computational cost and, hence, may be a burden in time-demanding applications. Thus, to improve the processing speed, we fix $P_{\mathrm{OP}}\left(F_{1}, F_{2}\right)$ as the average value computed from half of the database. The second assumption we made is that the overlapping is uniformly distributed across the feature map. Thus, we can use $\left(\left(d\left(F_{1}^{X}, F_{1}^{Y}\right)+\right.\right.$ $\left.\left.d\left(F_{2}^{X}, F_{2}^{Y}\right)\right) / 2\right) * P_{\mathrm{OP}}\left(F_{1}, F_{2}\right)$ as an approximation distance in the overlapping part.

By using (5), the distances between $X_{1}$ and $X_{2}$, and $X_{1}$ and $Y$ become 6.75 and 6 , respectively. It is much closer to the true distance than using (3). Similarly, we could extend the fusion scheme to fuse more bands, e.g., three spectral bands as in

$$
\begin{aligned}
& d_{F_{1} \cup F_{2} \cup F_{3}}(X, Y) \\
&= d\left(F_{1}^{X}, F_{1}^{Y}\right)+d\left(F_{2}^{X}, F_{2}^{Y}\right)+d\left(F_{3}^{X}, F_{3}^{Y}\right) \\
&-\frac{\left(d\left(F_{1}^{X}, F_{1}^{Y}\right)+d\left(F_{2}^{X}, F_{2}^{Y}\right)\right)}{2} * P_{\mathrm{OP}}\left(F_{1}, F_{2}\right) \\
&-\frac{\left(d\left(F_{1}^{X}, F_{1}^{Y}\right)+d\left(F_{3}^{X}, F_{3}^{Y}\right)\right)}{2} * P_{\mathrm{OP}}\left(F_{1}, F_{3}\right) \\
&-\frac{\left(d\left(F_{2}^{X}, F_{2}^{Y}\right)+d\left(F_{3}^{X}, F_{3}^{Y}\right)\right)}{2} * P_{\mathrm{OP}}\left(F_{2}, F_{3}\right) \\
&+\frac{\left(d\left(F_{1}^{X}, F_{1}^{Y}\right)+d\left(F_{2}^{X}, F_{2}^{Y}\right)+d\left(F_{3}^{X}, F_{3}^{Y}\right)\right)}{3} \\
& * P_{\mathrm{OP}}\left(F_{1}, F_{2}, F_{3}\right) .
\end{aligned}
$$

Because different bands highlight different features of the palm, these features may provide different discriminate capabilities. It is intuitive to use weighted sum as

$$
d_{\text {WeightSum }}=\sum_{i=1}^{n} w_{i} d_{i}
$$

where $w_{i}$ is the weight on $d_{i}$, the distance in the $i$ th band, and $n$ is the number of total bands. Equation (3) can be regarded as a special case of (7) when the weight is 1 for each spectrum. 
Using the reciprocal of the equal error rate (EER, a point when the false accept rate (FAR) is equal to the false reject rate (FRR)) as the weight is widely used in biometric systems [30]. If we take $d_{i}^{\prime}=w_{i} d_{i}$ as the normalized distance for band $i$, then we can extend our proposed score-level fusion scheme to weighted sum: multiply the original distance with the weight for normalization and then substitute the new distance into (5) or (6).

\section{EXPERIMENTAL RESULTS}

In this section, we report the experimental results on a large database. Section IV-A introduces the establishment of our multispectral palmprint database. Section IV-B reports the verification results on each band. Section IV-C reports the results using the proposed fusion scheme. Section IV-D shows some simple antispoofing tests. Finally, Section IV-E discusses the speed.

\section{A. Multispectral Palmprint Database $e^{1}$}

We collected multispectral palmprint images from 250 individuals using the developed data acquisition device. The subjects were mainly volunteers from our institute. In the database, 195 people are male, and the age distribution is from 20 to 60 years old. We collected the multispectral palmprint images on two separate sessions. The average time interval between the two occasions is 9 days. On each session, the subject was asked to provide six samples of each of his/her left and right palms. Therefore, our database contains 6000 images for each band from 500 different palms. For each shot, the device collected four images from the four bands (Red, Green, Blue, and NIR) in less than $1 \mathrm{~s}$. In palmprint acquisition, the users are asked to keep their palms stable on the device. The resolution of the images is $352 \times 288(<100 \mathrm{dpi})$.

\section{B. Palmprint Verification on Each Band}

As shown in Fig. 5, different bands can have different features of palm, providing different discriminate information for personal authentication. For different bands, different parameters should be used for better recognition results. This section is to investigate which spectral band works better for palmprint verification. Specifically, we try to find which band is preferred for palmprint verification via an experimental point of view.

In the following experiment, we set up a subset from the whole database by only using the first session images, totally 3000 images for each band. The subset is used for parameter selection in feature extraction. To obtain the verification accuracy, each palmprint image is matched with all the other palmprint images of the same band in the training database. A match is counted as a genuine matching if the two palmprint images are from the same palm; otherwise, the match is counted as impostor matching. The total number of matches is $3000 \times 2999 / 2=4498500$, and among them, there are 7500

\footnotetext{
${ }^{1}$ The database will shortly be available on the Biometrics Research Centre (BRC, www.comp.polyu.edu.hk/ biometrics) website.
}

TABLE $\mathrm{V}$

STATISTICS OF EER UNDER DIFFERENT PARAMETERS FOR DIFFERENT BANDS

\begin{tabular}{ccccc}
\hline \hline EER (\%) & Blue & Green & Red & NIR \\
\hline Mean & 0.0712 & 0.0641 & 0.0257 & 0.0430 \\
Std & 0.0142 & 0.0170 & 0.0217 & 0.0340 \\
Median & 0.0669 & 0.0666 & 0.0153 & 0.0378 \\
Minimal & 0.0400 & 0.0293 & 0.0015 & 0.0114 \\
Maximal & 0.1332 & 0.1607 & 0.1601 & 0.2665 \\
\hline \hline
\end{tabular}

$(6 \times 500 \times 5 / 2)$ genuine matching, and the others are impostor matching. The EER is used to evaluate the performance.

As stated in Section III, there are two variables for the Gabor filter: 1) $\kappa$ and 2) $\omega$. Different parameters may get different feature maps, as shown in Fig. 5. It is impossible to exhaustively search in the parameter space to find the optimal parameters for each band. We evaluate on 20 and 15 different values for $\kappa$ and $\omega$, respectively; thus, the total number of combination trial is 300. Here, the Gabor filter size is fixed as $35 \times 35$.

Some statistics of the EER are listed in Table V. Due to the limit of space, we do not show all the experiment results. Because of the different spectral characteristics of the different bands, the optimal (corresponding to minimal EER) parameters for different bands are different. Some findings could be found in Table V. First, the Red and NIR bands have better EER than the Blue and Green bands. This is mainly because Red and NIR could capture not only most of the palm line information but also some palm vein structures. This additional palm vein information helps classify those palms with similar palm lines. Fig. 10 shows an example: the two multispectral images of different palms have small distance under Blue [(a) and (e)] or Green [(b) and (f)] bands, which may lead to a false match; however, their distance under Red [(c) and (g)] or NIR [(d) and (h)] bands is large, which will lead to a correct match.

Second, the EER of NIR is higher than that of Red. There are mainly two reasons. First, the palm lines in the NIR band is not as strong as those in the Red band because NIR light can penetrate deeper the palm skin than Red light, which attenuates the reflectance. Second, some people, particularly females, have very weak vein structures under NIR light because their skin is a little thicker [24]. Fig. 11 shows an example. Fig. 11(b) and (d) shows the NIR images of a palm. The line structure in the two images is very weak compared with Fig. 11(a) and (c), which are the Red images of the palm. Meanwhile, the vein structure in Fig. 11(b) and (d) is not strong enough. Thus, the palm will falsely be recognized in the NIR band, whereas it can be recognized in the Red band. Third, the performance of the Blue and Green bands is very similar. As can be seen in Figs. 5 and 10, the palm lines in the Blue and Green bands look very similar. Last but not the least, the accuracy on shortwavelength bands (Blue and Green) is much more robust to parameter selection than that on long-wavelength bands (Red and NIR). One possible reason is that short-wavelength light scatters more than long-wavelength light [27], so palm lines are much thicker and clearer under short spectra.

We perform the palmprint verification on the whole database with the optimal parameters for each band. The same matching strategy is used as before. The numbers of genuine matching and impostor matching are 33000 and 17964000 , respectively. 


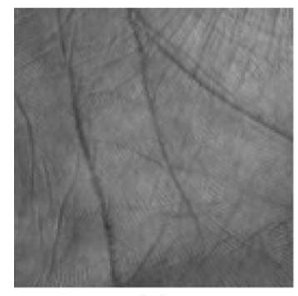

(a)

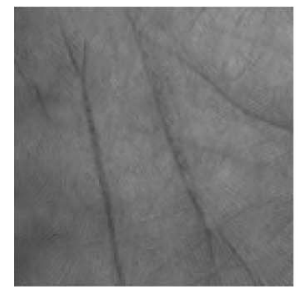

(e)

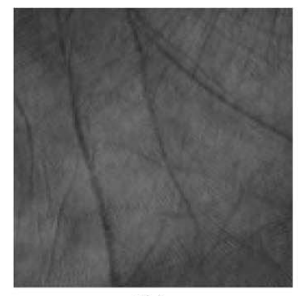

(b)

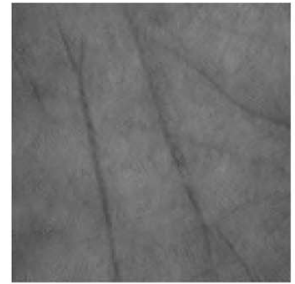

(f)

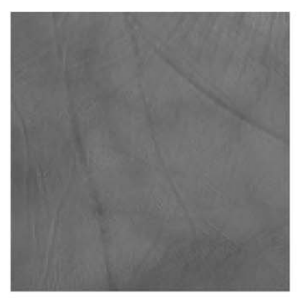

(c)

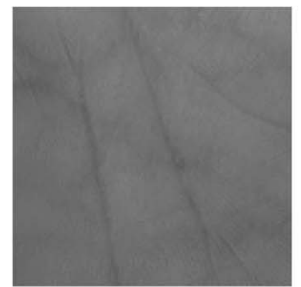

(g)

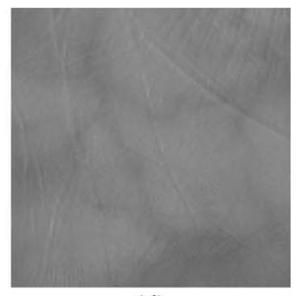

(d)

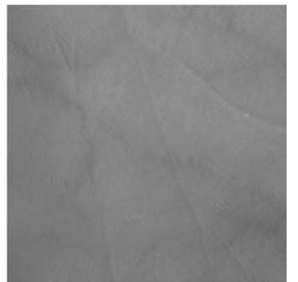

(h)

Fig. 10. Multispectral images of two different palms that are wrongly recognized under Blue or Green spectrum, but can correctly be recognized under Red or NIR spectrum. From (a)-(b) or (e)-(h), the four images correspond to Blue, Green, Red, and NIR, respectively.

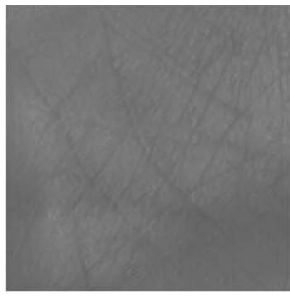

(a)

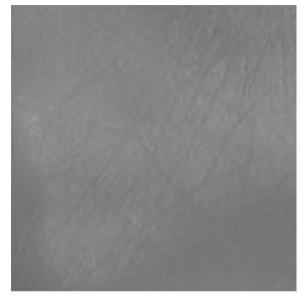

(b)

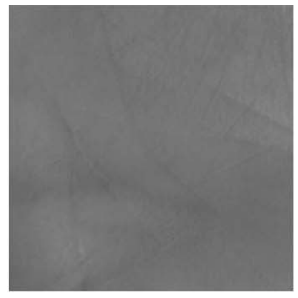

(c)

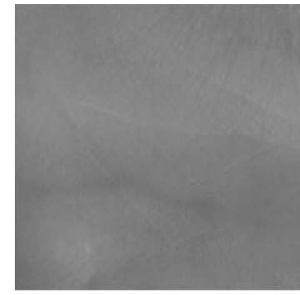

(d)

Fig. 11. Example of palm that is falsely recognized in NIR band but can correctly be recognized in the Red band. (a) and (c) Collected under Red light. (b) and (d) Collected under NIR light.

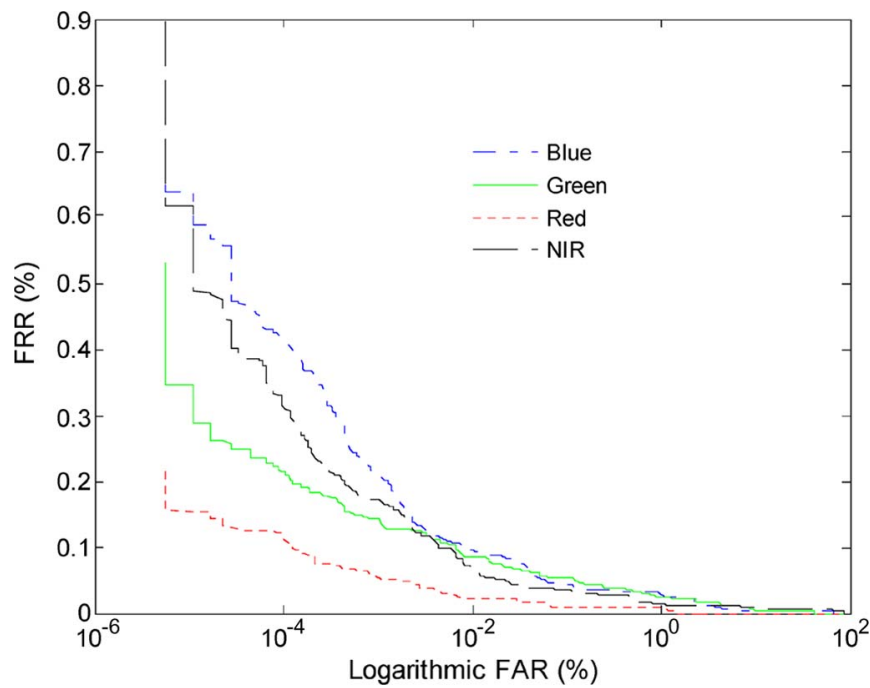

Fig. 12. Verification performance using optimal parameters for each spectrum.

The receiver operating characteristic curves for different spectral bands are shown in Fig. 12. The EER values for Blue, Green, Red, and NIR are $0.0520 \%, 0.0575 \%, 0.0212 \%$, and $0.0398 \%$, respectively. The accuracy on each single band is comparable with those of state-of-the-art (EER: 0.024\%) [32] on the public palmprint database [31] collected under white illumination.

\section{Palmprint Verification by Fusion}

The four bands can be fused to further improve the palmprint verification accuracy. Table VI lists the accuracy results by four different fusion schemes: 1) original weighted sum $(w=1)$; 2) proposed weighted sum $(w=1) ; 3)$ original weighted sum $(w=1 / \mathrm{EER})$; and 4$)$ proposed weighted sum $(w=1 / \mathrm{EER})$. Some findings could be obtained. First, all fusion schemes can result in smaller EER than a single band except the fusion of Blue and Green (this is because the feature overlapping between them is very high), which validates the effectiveness of multispectral palmprint authentication. Second, using the reciprocal of the EER as the weight usually leads to better results than the equal-weight scheme. Third, the proposed fusion scheme, which could reduce the feature overlapping effect, achieves better results than the original weighted-sum method. It can be verified that (5) can be rewritten as (7), and it is actually a weighted $\left(\left(1-P_{\mathrm{OP}}\left(F_{1}, F_{2}\right)\right)\right)$ distance of (3), e.g.,

$$
\begin{aligned}
d_{F_{1} \cup F_{2}}(X, Y)= & d\left(F_{1}\right)+d\left(F_{2}\right)-d\left(F_{1} \cap F_{2}\right) \\
= & d\left(F_{1}^{X}, F_{1}^{Y}\right)\left(1-P_{\mathrm{OP}}\left(F_{1}, F_{2}\right)\right) \\
& +d\left(F_{2}^{X}, F_{2}^{Y}\right)\left(1-P_{\mathrm{OP}}\left(F_{1}, F_{2}\right)\right) \\
= & \left(d\left(F_{1}^{X}, F_{1}^{Y}\right)+d\left(F_{2}^{X}, F_{2}^{Y}\right)\right) \\
& \times\left(1-P_{\mathrm{OP}}\left(F_{1}, F_{2}\right)\right) .
\end{aligned}
$$


TABLE VI

ACCuracy Measurement Comparison By DifFerent Fusion Schemes

\begin{tabular}{ccccc}
\hline EER (\%) & $\begin{array}{c}\text { Original Weighted } \\
\text { Sum }(\mathrm{w}=1)\end{array}$ & $\begin{array}{c}\text { Proposed } \\
\text { Weighted Sum } \\
(\mathrm{w}=1)\end{array}$ & $\begin{array}{c}\text { Original Weighted } \\
\text { Sum }(\mathrm{w}=1 / \mathrm{EER})\end{array}$ & $\begin{array}{c}\text { Proposed Weighted } \\
\text { Sum }(\mathrm{w}=1 / \text { EER })\end{array}$ \\
\hline Blue, Green & 0.0425 & 0.0425 & 0.0397 & 0.0397 \\
Blue, Red & 0.0154 & 0.0154 & $\mathbf{0 . 0 1 2 1}$ & $\mathbf{0 . 0 1 2 1}$ \\
Blue, NIR & 0.0212 & 0.0212 & 0.0212 & 0.0212 \\
Green, Red & 0.0212 & 0.0212 & 0.0182 & 0.0182 \\
Green, NIR & 0.0242 & 0.0242 & 0.0181 & 0.0181 \\
Red, NIR & 0.0152 & 0.0152 & 0.0152 & 0.0152 \\
Blue, Green, Red & 0.0243 & 0.0212 & 0.0152 & 0.0151 \\
Blue, Green, NIR & 0.0212 & 0.0214 & 0.0212 & 0.0212 \\
Blue, Red, NIR & $\mathbf{0 . 0 1 2 1}$ & $\mathbf{0 . 0 1 2 1}$ & $\mathbf{0 . 0 1 2 1}$ & $\mathbf{0 . 0 1 2 1}$ \\
Green, Red, NIR & 0.0153 & 0.0156 & 0.0152 & 0.0150 \\
Blue, Green, Red, NIR & 0.0152 & 0.0151 & $\mathbf{0 . 0 1 2 1}$ & $\mathbf{0 . 0 1 2 1}$ \\
\hline
\end{tabular}

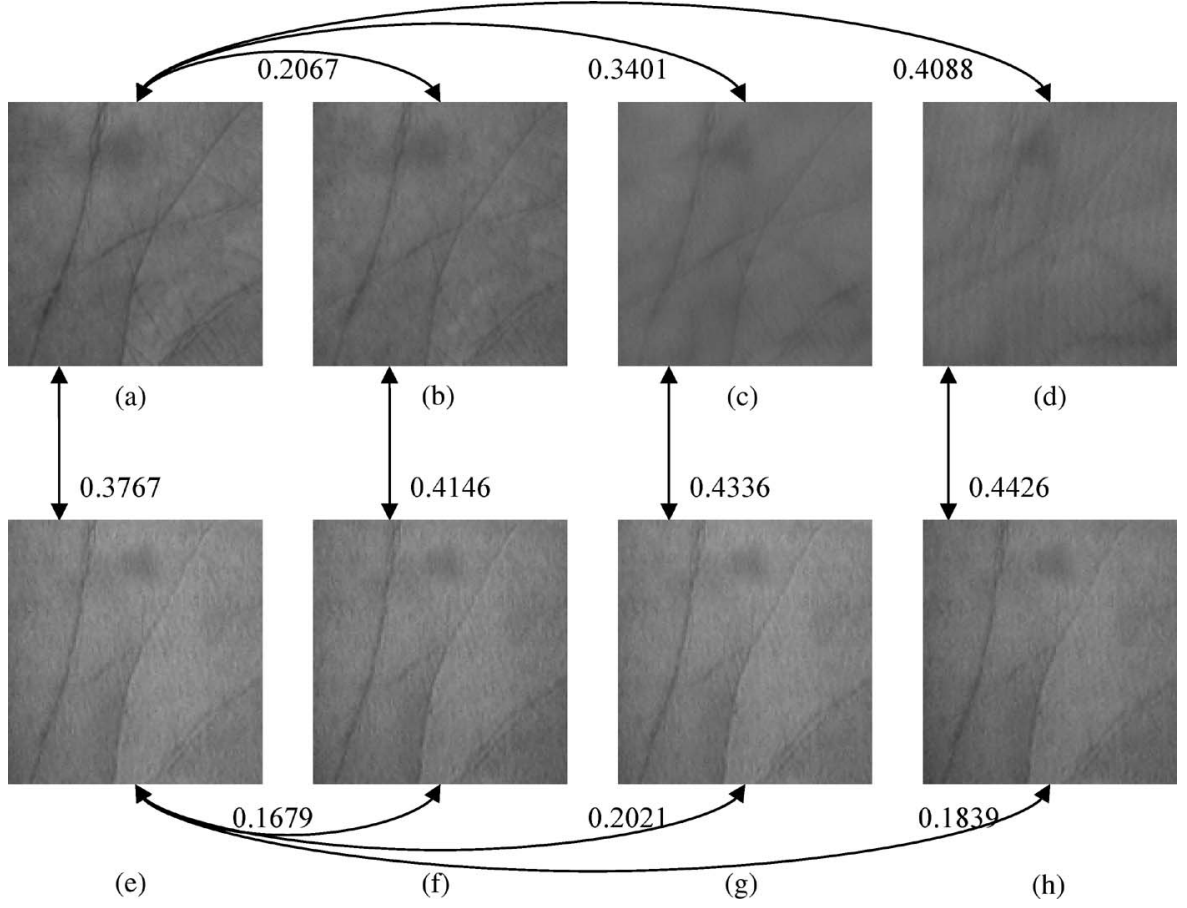

Fig. 13. Example of antispoof test. (a)-(d) Images of a true palm taken under Blue, Green, Red, and NIR. (e)-(h) Images of a fake palm taken under Blue, Green, Red, and NIR. The distance between two images is shown on or near a double arrow curve.

The best result is obtained by fusing Red and Blue bands, which leads an EER as low as $0.0121 \%$. To the best of our knowledge, our multispectral palmprint database (250 subjects) is the largest database so far. The numbers of subjects in the databases of [18]-[21] and [33] are 7, 50, 100, 120, and 165 , respectively. Among them, only the size of the database by Hao et al. [33] is close with ours. However, the best EER of their work is $0.50 \%$, which is much worse than ours $(0.0121 \%)$.

Although our fusion scheme verifies that the accuracy could be improved by fusing the features across spectral bands, sometimes the fusion of three or four bands is not better than the fusion of two bands. Certainly, it is possible that a better fusion scheme could be developed to more efficiently fuse the different features in different bands. How to further improve the fusion result will be explored in our future work.

\section{Antispoofing Test}

A good biometric system should be robust to spoof attacks. To test the antispoofing ability, a simple test is implemented. A palmprint image in Blue band is printed on a paper, and then this paper is used as a fake palm to attack the system. For comparison, we apply this test to both the single-spectral (i.e., the Blue channel) system and the multispectral system. As shown in Fig. 13, the faked palmprint is easy to pass the singlespectral system, and the distance (0.3767) between (a) and (e) is smaller than the mean impostor distance $(0.4621$, refer to Table II) and close to the verification threshold in our system. However, it is hard to pass the multispectral system because the distances in other bands are big. For example, the distance (0.4426) between (d) and (h) is close to the mean impostor distance $(0.4627)$ and far away from the verification threshold. Moreover, because the reflectance of the fake material 
TABLE VII

EXECUTION TIME

\begin{tabular}{cc}
\hline & Average Time (ms) \\
\hline Image Acquisition & $<1000$ \\
ROI Extraction & 138 \\
Feature Extraction & $36^{* 4}$ \\
Feature Matching & $0.06^{* 4}$ \\
\hline \hline
\end{tabular}

(paper in this example) is different from that of the skin, the distance between Blue and NIR bands of the faked palm is very small compared with that of the true palm (refer to Table II). Thus, this feature can be used for liveness detection to improve the robustness of our system.

\section{E. Speed}

The system is implemented using Visual $\mathrm{C}++6.0$ on a $\mathrm{PC}$ with Windows XP, T6400 CPU (2.13 GHz), and 2-GB random access memory. The execution time for each step is listed in Table VII. The total execution time of verification is less than $1.3 \mathrm{~s}$, which is fast enough for real-time application. As the speed of matching is fast, it can easily be extended to the identification system. For example, for 1-to-1000 identification, the total time cost is only $1.5 \mathrm{~s}$. By optimizing the program code, it is possible to further reduce the computational time.

\section{CONClusion}

In this paper, we have proposed an online multispectral palmprint system for real-time biometric authentication. Using palm line orientation code as features, we first evaluated palmprint verification using a single spectrum. It was verified that Red and NIR bands have better results than Blue and Green bands because they could capture not only palm line features but also palm vein features. Since the palm lines acquired from NIR are not as clear as those from Red, and the palm vein structure obtained from NIR solely is not discriminate enough, the NIR is a little inferior to Red. The Blue and Green bands have very similar results, and they are more robust to parameter selection than long-wavelength bands (Red and NIR bands).

Since different bands highlight different texture information, the fusion of them could significantly reduce the EER. It was found that the fusion of Red and Blue achieves the best result. A new score-level fusion scheme originating from the set theory was proposed to reduce the overlapping effect between bands. Experimental results show the effectiveness of our scheme. It was also found that due to the much redundant information across some bands, the fusion of three or four bands may not achieve better result than the fusion of two bands. How to better fuse the multispectral information will be investigated in the future.

\section{ACKNOWLEDGMENT}

The authors would like to thank the anonymous reviewers for their constructive comments.

\section{REFERENCES}

[1] A. Jain, R. Bolle, and S. Pankanti, Eds., Biometrics: Personal Identification in Network Society. Boston, MA: Kluwer, 1999.

[2] D. Zhang, Automated Biometrics-Technologies and Systems. Boston, MA: Kluwer, 2000.

[3] X. Wu, D. Zhang, and K. Wang, "Fisherpalm based palmprint recognition," Pattern Recognit. Lett., vol. 24, no. 15, pp. 2829-2838, Nov. 2003.

[4] T. Connie, A. T. B. Jin, and M. G. K. Ong, "An automated palmprint recognition system," Image Vis. Comput., vol. 23, no. 5, pp. 501-505, May 2005.

[5] D. Hu, G. Feng, and Z. Zhou, "Two-dimensional locality preserving projections (2DLPP) with its application to palmprint recognition," Pattern Recognit., vol. 40, no. 1, pp. 339-342, Jan. 2007.

[6] C. Han, H. Cheng, C. Lin, and K. Fan, "Personal authentication using palm-print features," Pattern Recognit., vol. 36, no. 2, pp. 371-381, Feb. 2003.

[7] X. Wu, D. Zhang, and K. Wang, "Palm line extraction and matching for personal authentication," IEEE Trans. Syst., Man, Cybern. A, Syst., Humans, vol. 36, no. 5, pp. 978-987, Sep. 2006.

[8] D. Zhang, W. Kong, J. You, and M. Wong, "Online palmprint identification," IEEE Trans. Pattern Anal. Mach. Intell., vol. 25, no. 9, pp. 10411050, Sep. 2003.

[9] A. Kong, D. Zhang, and M. Kamel, "Palmprint identification using feature-level fusion," Pattern Recognit., vol. 39, no. 3, pp. 478-487, Mar. 2006.

[10] A. Kong and D. Zhang, "Competitive coding scheme for palmprint verification," in Proc. Int. Conf. Pattern Recog., 2004, pp. 520-523.

[11] W. Jia, D.-S. Huang, and D. Zhang, "Palmprint verification based on robust line orientation code," Pattern Recognit., vol. 41, no. 5, pp. 15041513, May 2008

[12] S. Ribaric and I. Fratric, "A biometric identification system based on eigenpalm and eigenfinger features," IEEE Trans. Pattern Anal. Mach. Intell., vol. 27, no. 11, pp. 1698-1709, Nov. 2005.

[13] A. Kumar and D. Zhang, "Personal recognition using hand shape and texture," IEEE Trans. Image Process., vol. 15, no. 8, pp. 2454-2461, Aug. 2006

[14] Y.-F. Yao, X.-Y. Jing, and H.-S. Wong, "Face and palmprint feature level fusion for single sample biometrics recognition," Neurocomputing, vol. 70, no. 7-9, pp. 1582-1586, Mar. 2007.

[15] R. K. Rowe, K. A. Nixon, and S. P. Corcoran, "Multi spectral fingerprint biometrics," in Proc. Inf. Assurance Workshop, 2005, pp. 14-20.

[16] R. Singh, M. Vatsa, and A. Noore, "Hierarchical fusion of multispectral face images for improved recognition performance," Inf. Fusion, vol. 9, no. 2, pp. 200-210, Apr. 2008.

[17] J. H. Park and M. G. Kang, "Multispectral iris authentication system against counterfeit attack using gradient-based image fusion," Opt. Eng., vol. 46, no. 11, pp. 117003-1-117 003-14, Nov. 2007

[18] Y. Hao, Z. Sun, and T. Tan, "Comparative studies on multispectral palm image fusion for biometrics," in Proc. Asian Conf. Comput. Vis., 2007, pp. 12-21.

[19] R. K. Rowe, U. Uludag, M. Demirkus, S. Parthasaradhi, and A. K. Jain, "A multispectral whole-hand biometric authentication system," in Proc. Biometric Symp., Biometric Consortium Conf., Baltimore, MD, Sep. 2007, pp. 1-6.

[20] L. Likforman-Sulem, S. Salicetti, J. Dittmann, J. Ortega-Garcia, N. Pavesic, G. Gluhchev, S. Ribaric, and B. Sankur, Final report on the jointly executed research carried out on signature, hand and other modalities, BioSecure, Paris, France. [Online]. Available: http://biosecure. it - sudparis.eu/public_html/biosecure1/public_docs_deli/BioSecure_ Deliverable_D07-4-4_b2.pdf.pdf

[21] J.-G. Wang, W.-Y. Yau, A. Suwandy, and E. Sung, "Person recognition by fusing palmprint and palm vein images based on 'Laplacianpalm' representation," Pattern Recognit., vol. 41, no. 5, pp. 1514-1527, May 2008.

[22] D. J. Gawkrodger, Dermatology: An Illustrated Colour Text., 3rd ed. London, U.K.: Elsevier Health Sci., 2002.

[23] V. P. Zharov, S. Ferguson, J. F. Eidt, P. C. Howard, L. M. Fink, and M. Waner, "Infrared imaging of subcutaneous veins," Lasers Surg. Med., vol. 34, no. 1, pp. 56-61, Jan. 2004.

[24] Y. Lee and K. Hwang, "Skin thickness of Korean adults," Surg. Radiol. Anat., vol. 24, no. 3/4, pp. 183-189, Jan. 2002.

[25] A. A. Ross, K. Nadakumar, and A. K. Jain, Handbook of Multibiometrics. New York: Springer-Verlag, 2006.

[26] S. A. C. Schuckers, "Spoofing and anti-spoofing measures," Inf. Secur. Tech. Rep., vol. 7, no. 4, pp. 56-62, Dec. 2002.

[27] C. F. Bohren and D. Huffman, Absorption and Scattering of Light by Small Particles. New York: Wiley, 1983. 
[28] J. Daugman, "The importance of being random: Statistical principles of iris recognition," Pattern Recognit., vol. 36, no. 2, pp. 279-291, Feb. 2003.

[29] M. I. Sobel, Light. Chicago, IL: Univ. Chicago Press, 1989, pp. 52-62.

[30] R. Snelick, U. Uludag, A. Mink, M. Indovina, and A. Jain, "Large-scale evaluation of multimodal biometric authentication using state-of-the-art systems," IEEE Trans. Pattern Anal. Mach. Intell., vol. 27, no. 3, pp. 450455, Mar. 2005.

[31] PolyU Palmprint Database. [Online]. Available: http://www.comp.polyu. edu.hk/ biometrics

[32] W. Zuo, F. Yue, K. Wang, and D. Zhang, "Multiscale competitive code for efficient palmprint recognition," in Proc. Int. Conf. Pattern Recog., 2008, pp. 1-4.

[33] Y. Hao, Z. Sun, T. Tan, and C. Ren, "Multispectral palm image fusion for accurate contact-free palmprint recognition," in Proc. Int. Conf. Image Process., 2008, pp. 281-284.

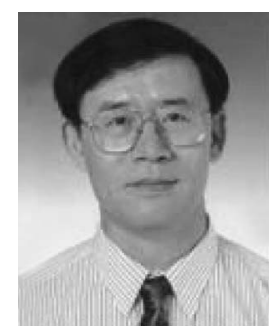

David Zhang (F'09) received the degree in computer science from Peking University, Beijing, China, the M.Sc. degree in computer science and the Ph.D. degree from Harbin Institute of Technology (HIT), Harbin, China, in 1982 and 1985, respectively, and the Ph.D. degree in electrical and computer engineering from the University of Waterloo, Ontario, Canada, in 1994.

From 1986 to 1988, he was a Postdoctoral Fellow with Tsinghua University, Beijing, and then an Associate Professor with the Academia Sinica, Beijing. He is currently the Head of the Department of Computing and a Chair Professor with the Hong Kong Polytechnic University, Hong Kong, where he is the Founding Director of the Biometrics Technology Centre (UGC/CRC) supported by the Hong Kong SAR Government in 1998. He also serves as a Visiting Chair Professor with Tsinghua University and an Adjunct Professor with Peking University; Shanghai Jiao Tong University, Shanghai, China; HIT; and the University of Waterloo. He is the Founder and Editor-in-Chief for the International Journal of Image and Graphics, a Book Editor for the Springer International Series on Biometrics (KISB), and an Associate Editor for more than ten international journals, including IEEE TRANSACTIONS and Pattern Recognition. He is the author of more than ten books and 200 journal papers.

Prof. Zhang is a Croucher Senior Research Fellow, a Distinguished Speaker of the IEEE Computer Society, and a Fellow of the International Association of Pattern Recognition. He is the Organizer of the International Conference on Biometrics Authentication.

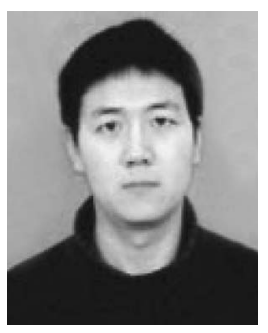

Zhenhua Guo received the B.S. and M.S. degrees in computer science from Harbin Institute of Technology, Harbin, China, in 2002 and 2004, respectively. $\mathrm{He}$ is currently working toward the Ph.D. degree with the Department of Computing, The Hong Kong Polytechnic University, Kowloon, Hong Kong.

From 2005 to 2007, he was a Research Assistant with the Department of Computing, The Hong Kong Polytechnic University. His research interests include pattern recognition, texture classification, biometrics, etc.

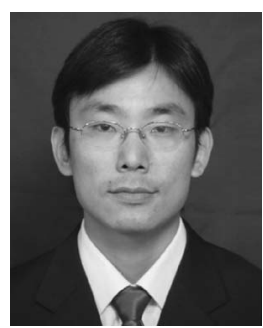

Guangming Lu received the B.S., M.S., and Ph.D. degrees from Harbin Institute of Technology, Harbin, China, in 1998, 2000, and 2005, respectively.

From 2005 to 2007, he was a Postdoctoral Fellow with Tsinghua University, Beijing, China. Since 2007, he has been an Associate Professor with the Shenzhen Graduate School, Harbin Institute of Technology, Shenzhen, China. His research interests include image processing, pattern recognition, biometrics, etc.

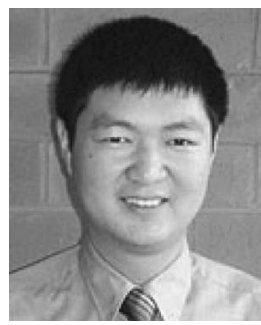

Lei Zhang (M'04) received the B.S. degree from Shenyang Institute of Aeronautical Engineering, Shenyang, China, in 1995 and the M.S. and Ph.D. degrees in control theory and applications from Northwestern Polytechnical University, Xi'an, China, in 1998 and 2001, respectively.

From 2001 to 2002, he was a Research Associate with the Department of Computing, The Hong Kong Polytechnic University, Kowloon, Hong Kong. From January 2003 to January 2006, he was a Postdoctoral Fellow with the Department of Electrical and Computer Engineering, McMaster University, Hamilton, ON, Canada. Since January 2006, he has been an Assistant Professor with the Department of Computing, The Hong Kong Polytechnic University. His research interests include image and video processing, biometrics, pattern recognition, multisensor data fusion, optimal estimation theory, etc.

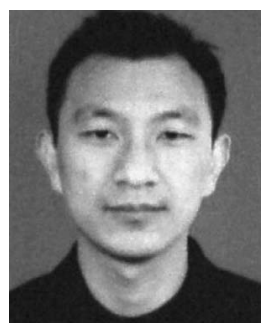

Wangmeng Zuo received the Ph.D. degree in computer science and technology from Harbin Institute of Technology, Harbin, China, in 2007.

$\mathrm{He}$ is currently a Lecturer with the School of Computer Science and Technology, Harbin Institute of Technology. He is the author of 20 scientific papers in pattern recognition and computer vision. His current research interests include pattern recognition, computer vision, and their applications in biometrics, medical diagnosis, and bioinformatics.

Dr. Zuo is a Reviewer of several international journals, including IEEE TRANSACTIONS ON INFORMATION FORENSICS AND SECURITY, IEEE TRANSACTIONS ON InSTRUMENTATION AND MEASUREMENT, IET Signal Processing, Neurocomputing, and International Journal of Image and Graphics. 